\title{
Effective surface properties of light, heavy, and superheavy nuclei
}

\author{
Abdul Quddus ${ }^{1}$, M. Bhuyan ${ }^{2,3}$, and S. K. Patra ${ }^{4,5}$ \\ ${ }^{1}$ Department of Physics, Aligarh Muslim University, Aligarh-202002, India \\ ${ }^{2}$ Department of Physics, Faculty of Science, University of Malaya, Kuala Lumpur \\ 50603, Malaysia \\ ${ }^{3}$ Institute of Research Development, Duy Tan University, Da Nang 550000 Vietnam \\ ${ }^{4}$ Institute of Physics, Bhubaneswar-751005, India \\ ${ }^{5}$ Homi Bhabha National Institute, Training School Complex, Anushakti Nagar, \\ Mumbai 400085, India
}

\begin{abstract}
Starting from light to superheavy nuclei, we have calculated the effective surface properties such as the symmetry energy, neutron pressure, and symmetry energy curvature using the coherent density fluctuation model. The isotopic chains of $\mathrm{O}, \mathrm{Ca}, \mathrm{Ni}, \mathrm{Zr}, \mathrm{Sn}, \mathrm{Pb}$, and $\mathrm{Z}=120$ are considered in the present analysis, which cover nuclei over the whole nuclear chart. The matter density distributions of these nuclei along with the ground state bulk properties are calculated within the spherically symmetric effective field theory motivated relativistic mean field model by using the recently developed IOPB-I, FSUGarnet, and G3 parameter sets. The calculated results are compared with the predictions of the widely used NL3 parameter set and found in good agreement. We observe a few signatures of shell and/or sub-shell structure in the isotopic chains of nuclei. The present investigations are quite relevant for the synthesis of exotic nuclei with high isospin asymmetry including superheavy and also to constrain an equation of state of nuclear matter.
\end{abstract}

PACS numbers: 21.10.-k, 21.10.Gv, 21.65.-f, 21.65.Mn 


\section{Introduction}

Nuclei lie away from $\beta$ - stability line with large neutron to proton asymmetry are of great importance. One of the quests among the nuclear physics community is how to synthesis the exotic and superheavy nuclei and to explore their applications. About 3000 nuclei, lie away from the $\beta$-stable line, have been synthesized in various laboratories and some more $(\sim 5000)$ have to be synthesized. Since the matter at extreme density and temperature is impossible to create in a laboratory, a study of neutron-rich nuclei is treated as a tool to understand it. In the quest for the formation of superheavy nuclei, the last one with $Z=118$ has been synthesized at Dubna which was named Oganesson [1] and more superheavy nuclei are expected to synthesize. A lot of theoretical predictions are reported about the stability of superheavy nuclei against the spontaneous fission, $\alpha$ - and $\beta$-decays, and neutron emission [2,3]. The mere existence of exotic nuclei including superheavy is entirely by the quantal shell effects, which play against the surface tension and the Coulomb repulsion. Furthermore, one of the very compelling issue in such exotic systems is the appearance of new magic numbers and the disappearance of others in moving from $\beta$-stable to drip-line region of the nuclear chart $[4,5]$. For example, beyond the proton number $Z=82$ and neutron number $N=126$, the next predicted magic numbers are $\mathrm{Z}=114,120$, and 126 for the proton and $\mathrm{N}=172$ or 184 for the neutron [3]. The neutron-rich/deficient isotopes, and $\mathrm{Z}=$ 120 element which is one of the predicted magic numbers represent a challenge for future experimental synthesis since they are located at the limit of accessibility with available cold fusion reactions facility. Therefore, an accurate estimation of their characteristics are essential from the theoretical side to guide future experiment. Various experiments around the globe like Jyaväskylä (Finland) [6], FRIB (US) [7], GSI (Germany) [8], RIKEN (Japan) [9], GANIL (France) [10], FLNR (Russia) [11], CSR (China) [12], FAIR (Germany) [13], and ORNL (US) [14] provide a possibility of exploring an exotic nuclei, and superheavy nuclei under extreme condition of isospin asymmetry.

The nuclear symmetry energy is directly connected with the isospin asymmetry of the system; either infinite nuclear matter or finite nuclear system. It is an important quantity having significant role in different areas of nuclear physics, for example, in structure of ground state nuclei $[15,16,17]$, physics of giant collective excitation [18], dynamics of heavy-ion reactions [19, 20], and physics of neutron star [21, 22, 23, 24]. It determines various neutron star properties such as mass-radius trajectory, its cooling rates, the thickness of the crust, and the moment of inertia [25]. The astrophysical observations and availability of exotic beam in a laboratory have raised an interest in symmetry energy [26]. In the last three decades, the density dependence of nuclear symmetry energy has played a great role to understand nuclei near the drip-line [27]. For interpreting the neutron-rich nuclei and the neutron star matter, the characterization of the symmetry energy through experiments is a crucial step. But, the symmetry energy is not a directly measurable quantity. It is extracted from the observables related to it. Danielewicz has demonstrated that the ratio of the bulk symmetry energy to the surface 
symmetry energy is related to the neutron skin-thickness [28]. It is found that the radius of a neutron star is correlated to the density dependence of the symmetry energy at a saturation point [29]. Furthermore, the $L$ coefficient (or say, pressure $P$ ) is correlated with the neutron skin-thickness of ${ }^{208} \mathrm{~Pb}[30,31,32,33]$ and the radius of a neutron star. Even the precise measurement of neutron skin-thickness is difficult, yet [34] it is one of the sensitive probes for nuclear symmetry energy. In some of our previous works as well as the works of others $[26,35]$, it has been shown that the symmetry energy of finite nuclei can be used as an observable to indicate/determine magic nuclei within Coherent density fluctuation model.

The neutron pressure of finite nuclei is related to the slope parameter $(L-)$ of symmetry energy at saturation, which is an essential quantity in determining the equation of state (EoS) of nuclear matter [26, 35, 36, 37]. Furthermore, for finite nuclei, the pressure depends on the strength of interaction among nucleons and their distributions. In our previous work [37], we have shown a correlation between neutron pressure and the neutron skin-thickness of neutron-rich thermally fissile nuclei. The symmetry energy and neutron pressure are collectively termed as effective surface properties, which are extensively defined in Refs. [26, 35] and also illustrated in Subsec. 3.2. The importance of the surface properties and their sensitivity to density have motivated us to pursue their systematic study over all regions of the nuclear chart. Here, we have investigated the effective surface properties for the isotopic series of $\mathrm{O}, \mathrm{Ca}, \mathrm{Ni}$, $\mathrm{Zr}, \mathrm{Sn}, \mathrm{Pb}$, and $\mathrm{Z}=120$ nuclei, which cover all the region of the mass table from light to superheavy region. Recently, the symmetry energy of finite nuclei at a local density has been studied by using various formulae of the liquid drop model [38, 39, 40], the energy density functional of Skyrme force [41, 42, 43], the random phase approximation based on the Hartree-Fock (HF) approach [44], the relativistic nucleon-nucleon interaction $[45,46]$, and the effective relativistic Lagrangian with density-dependent meson-nucleon vertex function [47]. In Refs. [26, 35, 48, 49] the surface properties of the nuclei have been studied by folding the nuclear matter properties, within the Brueckner energy density functional [50, 51], with the weight functions of the nuclei in the coherent density fluctuation model (CDFM) [48, 52]. The advantages of CDFM over other methods are that this method takes care (i) the fluctuation arises in the nuclear density distribution via weight function $|f(x)|^{2}$, and (ii) the momentum distributions through the mixed density matrix (i.e., the Wigner distribution function) $[35,26,49]$. In other words, the CDFM approach is adept to comprise the variation arise from the density and momentum distributions at the surface of finite nuclei. The present investigation covers systematic studies of the effective surface properties of several nuclei over the nuclear chart by finding the bulk properties along with densities of the nuclei within the effective field theory motivated relativistic mean field (E-RMF) approach. The calculated densities of the nuclei are served as the input to the CDFM to investigate the surface properties.

The paper is organized as follows: in Sec. 2 we present the formalism followed to carry out this work. In Sub-section 2.1, we outline effective field theory motivated 
relativistic mean field model, which has been used to calculate the ground state bulk properties and densities of the nuclei. Sub-section 2.2 contains the general idea of calculating symmetry energy and relevant quantities like pressure and symmetry energy curvature. In Sub-section 2.3 we present the formalism (CDFM) to calculate the effective surface properties. The calculated results are discussed in Sec. 3. Finally, the work is summarized in Sec. 4.

\section{Formalism}

\subsection{Effective field theory motivated relativistic mean field model (E-RMF)}

Relativistic mean field (RMF) theory is one of the microscopic approaches to solve the many body problem of nuclear system. In the RMF model, the nucleons are assumed to interact through the exchange of mesons. The model predicts ground as well as an intrinsic excited state properties of nuclei such as the binding energy, root mean square (RMS) radius, nuclear density distributions, deformation parameter, and single particle energies throughout the nuclear landscape. The details of the RMF models and their parameterizations can be found in Refs. [53, 54, 55, 56, 57, 58, 59]. For the sake of completeness, here we present the E-RMF formalism briefly. Effective mean field approximated Lagrangian density has, in principle, several numbers of terms with all possible types of self and cross-couplings of mesons. To handle E-RMF numerically, the ratios of fields and the nucleon mass are used in the truncation scheme as a constrain of naturalness. In this work, we have used the E-RMF Lagrangian having contributions of $\delta$ - meson and photon up to $2^{\text {th }}$ order exponent and the rest up to $4^{\text {th }}$ order of exponents, which has been shown to be reasonably good approximation to predict the finite nuclei and the nuclear matter observables up to considerable satisfaction [58]. The energy density, obtained within the E-RMF Lagrangian by applying mean field approximation, is given as:

$$
\begin{aligned}
\mathcal{E}(r)= & \sum_{i} \varphi_{i}^{\dagger}(r)\left\{-i \boldsymbol{\alpha} \cdot \boldsymbol{\nabla}+\beta\left[M-\Phi(r)-\tau_{3} D(r)\right]+W(r)+\frac{1}{2} \tau_{3} R(r)\right. \\
& \left.+\frac{1+\tau_{3}}{2} A(r)-\frac{i \beta \boldsymbol{\alpha}}{2 M} \cdot\left(f_{\omega} \boldsymbol{\nabla} W(r)+\frac{1}{2} f_{\rho} \tau_{3} \boldsymbol{\nabla} R(r)\right)\right\} \varphi_{i}(r) \\
& +\left(\frac{1}{2}+\frac{\kappa_{3}}{3 !} \frac{\Phi(r)}{M}+\frac{\kappa_{4}}{4 !} \frac{\Phi^{2}(r)}{M^{2}}\right) \frac{m_{s}^{2}}{g_{s}^{2}} \Phi^{2}(r)-\frac{\zeta_{0}}{4 !} \frac{1}{g_{\omega}^{2}} W^{4}(r) \\
& +\frac{1}{2 g_{s}^{2}}\left(1+\alpha_{1} \frac{\Phi(r)}{M}\right)(\boldsymbol{\nabla} \Phi(r))^{2}-\frac{1}{2 g_{\omega}^{2}}\left(1+\alpha_{2} \frac{\Phi(r)}{M}\right)(\boldsymbol{\nabla} W(r))^{2} \\
& -\frac{1}{2}\left(1+\eta_{1} \frac{\Phi(r)}{M}+\frac{\eta_{2}}{2} \frac{\Phi^{2}(r)}{M^{2}}\right) \frac{m_{\omega}^{2}}{g_{\omega}^{2}} W^{2}(r)-\frac{1}{2 e^{2}}(\nabla A(r))^{2}
\end{aligned}
$$




$$
\begin{aligned}
& -\frac{1}{2 g_{\rho}^{2}}(\nabla R(r))^{2}-\frac{1}{2}\left(1+\eta_{\rho} \frac{\Phi(r)}{M}\right) \frac{m_{\rho}^{2}}{g_{\rho}^{2}} R^{2}(r)-\Lambda_{\omega}\left(R^{2}(r) \times W^{2}(r)\right) \\
& +\frac{1}{2 g_{\delta}^{2}}(\nabla D(r))^{2}+\frac{1}{2} \frac{m_{\delta}^{2}}{g_{\delta}^{2}}\left(D^{2}(r)\right),
\end{aligned}
$$

where $\Phi, W, R, D$ and $A$ are the fields which have been redefined as $\phi=g_{\sigma} \sigma, W=g_{\omega} \omega^{0}$, $R=g_{\rho} \rho^{0}$, and $A=e A^{0} . m_{\sigma}, m_{\omega}, m_{\rho}$ and $m_{\delta}$ are the masses and $g_{\sigma}, g_{\omega}, g_{\rho}, g_{\delta}, \frac{e^{2}}{4 \pi}$ are the coupling constants for $\sigma, \omega, \rho, \delta$ mesons and photon, respectively. Using the EulerLagrangian equation of motion to Eq. 1, we get two first-order coupled differential equations for nucleons and four second-order differential equations for the four types of the meson fields [57]. The detailed equations can be found in Refs. [57, 58]. We transformed the Dirac equation into a Shrödinger-like form as it is done in [57] by eliminating the smaller component of the Dirac spinor. Then the equation is solved by following the procedure of Vautherin and Brink [60] with a fourth-order Runge-Kutta algorithm and the meson fields are solved by the Newton-Raphson method [54, 57, 58].

The total energy of a nucleus is given by following expression:

$$
E=\int \mathcal{E}(r) d^{3} r+E_{c m}+E_{\text {pair }} ;
$$

where, first term has the contribution of mesonic and nucleonic energy densities given by Eq. 1. The second and third terms are the centre-of-mass correction energy and pairing energy, respectively. The expression for $E_{c m}$ is given as:

$$
E_{c m}=-\frac{3}{4} \times 41 A^{-1 / 3} .
$$

To describe open-shell nuclei (other than double magic nuclei), certainly pairing plays a crucial role. In general, the BCS approximation with a constant gap/force scheme is adopted on top of the RMF/E-RMF formalism to take care of the pairing correlation. However, this prescription does not hold good for drip-line nuclei as the seniority pairing recipe fails for such exotic nuclei. This is because the coupling to the continuum in the normal BCS approximation is not taken correctly. This deficiency can be removed to some extent by including few quasi-bound states owing to their centrifugal barrier [61]. These quasi-bound states mock up the influence of the continuum. Here, we follow the procedure of Refs. [54]. For drip-line nuclei, there are no bound singleparticle levels above the Fermi surface. In our calculations, we take the bound-state contributions and the levels coming from the quasi-bound states at positive energies [61] and the expressions for $E_{\text {pair }}$ is written as:

$$
E_{\text {pair }}=-\frac{\Delta_{i}^{2}}{G_{i}}
$$

where $\Delta_{i}$ and $G_{i}\left(=C_{i} / A\right)$ are, respectively, the pairing gap and strength with $i=n, p$. The $C_{i}$ are chosen in a way to reproduce the binding energy of a nucleus with mass number $A$. For G2 set, $C_{n}=21$ and $C_{p}=22.5 \mathrm{MeV}$, and for FSUGarnet, G3 and IOPB-I sets, $C_{n}=19$ and $C_{p}=21 \mathrm{MeV}$. 


\subsection{The key equation of state parameters in nuclear matter}

The total energy of a nucleus given in the liquid droplet model, which is an extension of the Bethe Weizscker liquid drop model incorporating the volume and surface asymmetry is expressed as [21, 38]:

$$
\begin{aligned}
E(A, Z)= & -B . A+E_{S} A^{2 / 3}+S_{V} A \frac{(1-2 Z / A)^{2}}{\left(1+S_{S} A^{-1 / 3} / S_{V}\right)}+E_{C} \frac{Z^{2}}{A^{1 / 3}} \\
& +E_{d i f} \frac{Z^{2}}{A}+E_{e x} \frac{Z^{4 / 3}}{A^{1 / 3}}+a \Delta A^{-1 / 2} .
\end{aligned}
$$

Where $B \sim 16 \mathrm{MeV}$ is the binding energy per nucleon of bulk symmetric matter at saturation. $E_{S}, E_{C}, E_{d i f}$, and $E_{e x}$ are the coefficients for the surface energy of symmetric matter, the Coulomb energy of a uniformly charged sphere, the diffuseness correction, and the exchange correction to the Coulomb energy, respectively. The last term gives the pairing corrections, which is essential for open-shell nuclei. $S_{V}$ is the volume symmetry energy parameter and $S_{S}$ is the modified surface symmetry energy parameter in the liquid model (see Ref. [21]). The third term on the right-hand side of the above equation is the contribution of the symmetry energy to the total energy of a nucleus which can not be neglected and has a significant impact where N/Z ratio widely differs from the value at the valley of stability (drip-line nuclei).

Moreover, the energy per nucleon of nuclear matter $\mathcal{E} / A=e(\rho, \alpha)$ (where $\rho$ is the baryon density) can be expanded by Taylor series expansion method in terms of isospin asymmetry parameter $\alpha\left(=\frac{\rho_{n}-\rho_{p}}{\rho_{n}+\rho_{p}}\right)$ :

$$
e(\rho, \alpha)=\frac{\mathcal{E}}{\rho_{B}}-M=e(\rho)+S(\rho) \alpha^{2}+\mathcal{O}\left(\alpha^{4}\right),
$$

where $e(\rho), S(\rho)$ and $M$ are the energy density of symmetric nuclear matter (SNM) ( $\alpha$ $=0$ ), the symmetry energy, and the mass of a nucleon, respectively. The odd powers of $\alpha$ are forbidden by the isospin symmetry and the terms proportional to $\alpha^{4}$ and higher orders have a negligible contribution. The symmetry energy $S(\rho)$ is defined by:

$$
S(\rho)=\frac{1}{2}\left[\frac{\partial^{2} e(\rho, \alpha)}{\partial \alpha^{2}}\right]_{\alpha=0} .
$$

Near the saturation density $\rho_{0}$, the symmetry energy can be expanded through the Taylor series expansion method as:

$$
S(\rho)=J+L \mathcal{Y}+\frac{1}{2} K_{\text {sym }} \mathcal{Y}^{2}+\frac{1}{6} Q_{\text {sym }} \mathcal{Y}^{3}+\mathcal{O}\left[\mathcal{Y}^{4}\right],
$$

where $J=S\left(\rho_{0}\right)$ is the symmetry energy at saturation and $\mathcal{Y}=\frac{\rho-\rho_{0}}{3 \rho_{0}}$. The slope parameter $\left(L-\right.$ coefficient), the symmetry energy curvature $\left(K_{\text {sym }}\right)$, and the skewness parameter $\left(Q_{\text {sym }}\right)$ are defined as:

$$
\begin{aligned}
& L=\left.3 \rho \frac{\partial S(\rho)}{\partial \rho}\right|_{\rho=\rho_{0}}, \\
& K_{\text {sym }}=\left.9 \rho^{2} \frac{\partial^{2} S(\rho)}{\partial \rho^{2}}\right|_{\rho=\rho_{0}},
\end{aligned}
$$




$$
Q_{\text {sym }}=\left.27 \rho^{3} \frac{\partial^{3} S(\rho)}{\partial \rho^{3}}\right|_{\rho=\rho_{0}}
$$

respectively. The neutron pressure of asymmetric nuclear matter can also be evaluated from the slope parameter $(L-)$ by using the relation:

$$
L^{N M}=\frac{3 P_{0}^{N M}}{\rho_{0}} .
$$

It is to note that these nuclear matter properties at saturation (i.e., $\rho_{0}, S\left(\rho_{0}\right), L\left(\rho_{0}\right)$, $K_{\text {sym }}\left(\rho_{0}\right)$, and $\left.Q_{\text {sym }}\left(\rho_{0}\right)\right)$ are model dependent and vary with certain uncertainties. More details of these quantities and their values along with the allowed ranges for the nonrelativistic and relativistic mean field models with various force parameters can be found in Refs. [23, 24].

\subsection{The coherent density fluctuation model (CDFM)}

The CDFM was suggested and developed in Refs. [48, 52]. In the CDFM, the one-body density matrix $\rho\left(\mathbf{r}, \mathbf{r}^{\prime}\right)$ of a nucleus can be written as a coherent superposition of infinite number of one-body density matrices $\rho_{x}\left(\mathbf{r}, \mathbf{r}^{\prime}\right)$ for spherical pieces of the nuclear matter called as Fluctons [26, 35, 48],

$$
\rho_{x}(\mathbf{r})=\rho_{0}(x) \Theta(x-|\mathbf{r}|),
$$

with $\rho_{o}(x)=\frac{3 A}{4 \pi x^{3}}$. The generator coordinate $x$ is the spherical radius of the nucleus contained in a uniformly distributed spherical Fermi gas. In finite nuclear system, the one body density matrix can be given as [26, 35, 48, 49],

$$
\rho\left(\mathbf{r}, \mathbf{r}^{\prime}\right)=\int_{0}^{\infty} d x|f(x)|^{2} \rho_{x}\left(\mathbf{r}, \mathbf{r}^{\prime}\right),
$$

where, $|f(x)|^{2}$ is the weight function (Eq. (18)). The term $\rho_{x}\left(\mathbf{r}, \mathbf{r}^{\prime}\right)$ is the coherent superposition of the one body density matrix and defined as,

$$
\rho_{x}\left(\mathbf{r}, \mathbf{r}^{\prime}\right)=3 \rho_{0}(x) \frac{J_{1}\left(k_{f}(x)\left|\mathbf{r}-\mathbf{r}^{\prime}\right|\right)}{\left(k_{f}(x)\left|\mathbf{r}-\mathbf{r}^{\prime}\right|\right)} \times \Theta\left(x-\frac{\left|\mathbf{r}+\mathbf{r}^{\prime}\right|}{2}\right) .
$$

Here, $\mathrm{J}_{1}$ is the first order spherical Bessel function. The Fermi momentum of nucleons in the Fluctons with radius $x$ is expressed as $k_{f}(x)=\left(3 \pi^{2} / 2 \rho_{0}(x)\right)^{1 / 3}=\gamma / x$, where $\gamma=(9 \pi A / 8)^{1 / 3} \approx 1.52 A^{1 / 3}$. The Wigner distribution function of the one body density matrices in Eq. (15) is,

$$
W(\mathbf{r}, \mathbf{k})=\int_{0}^{\infty} d x|f(x)|^{2} W_{x}(\mathbf{r}, \mathbf{k})
$$

Here, $W_{x}(\mathbf{r}, \mathbf{k})=\frac{4}{8 \pi^{3}} \Theta(x-|\mathbf{r}|) \Theta\left(k_{F}(x)-|\mathbf{k}|\right)$. Similarly, the density $\rho(\mathrm{r})$ within CDFM can express in terms of the same weight function as,

$$
\rho(r)=\int d \mathbf{k} W(\mathbf{r}, \mathbf{k})=\int_{0}^{\infty} d x|f(x)|^{2} \frac{3 A}{4 \pi x^{3}} \Theta(x-|\mathbf{r}|)
$$

and it is normalized to the nucleon numbers of the nucleus, $\int \rho(\mathbf{r}) d \mathbf{r}=A$. By taking the $\delta$-function approximation to the Hill-Wheeler integral equation, we can obtain the 
differential equation for the weight function in the generator coordinate [48, 35]. The weight function for a given density distribution $\rho(\mathrm{r})$ can be expressed as,

$$
|f(x)|^{2}=-\left(\frac{1}{\rho_{0}(x)} \frac{d \rho(r)}{d r}\right)_{r=x},
$$

with $\int_{0}^{\infty} d x|f(x)|^{2}=1$. For a detailed analytical derivation, one can follows Refs. $[35,62,63]$. The symmetry energy, neutron pressure, and symmetry energy curvature for a finite nucleus are defined below by weighting the corresponding quantities for the infinite nuclear matter within the CDFM. The CDFM allows us to make a transition from the properties of nuclear matter to those of finite nuclei. Following the CDFM approach, the expression for the effective symmetry energy $S$, pressure $P$, and curvature $\Delta K$ for a nucleus can be written as $[26,35,49,62,63,64]$,

$$
\begin{aligned}
& S=\int_{0}^{\infty} d x|f(x)|^{2} S_{0}^{N M}(\rho(x)), \\
& P=\int_{0}^{\infty} d x|f(x)|^{2} P_{0}^{N M}(\rho(x)), \\
& \Delta K=\int_{0}^{\infty} d x|f(x)|^{2} \Delta K_{0}^{N M}(\rho(x)) .
\end{aligned}
$$

Here, the quantities on the left-hand-side of Eqs. (19-21) are the surface weighted average of the corresponding nuclear matter quantities with local density approximation, which have been determined within the method of Brueckner et. al., [50, 51].

In the present work considering the pieces of nuclear matter with density $\rho_{0}(\mathrm{x})$, we have used the matrix element $\mathrm{V}(\mathrm{x})$ of the nuclear Hamiltonian the corresponding energy of nuclear matter from the method of Brueckner et. al., [50, 51]. In Brueckner energy density functional method, the $\mathrm{V}(\mathrm{x})$ is given by:

$$
V(x)=A V_{0}(x)+V_{C}+V_{C O},
$$

where

$$
\begin{aligned}
& V_{0}(x)=37.53\left[(1+\delta)^{5 / 3}+(1-\delta)^{5 / 3}\right] \rho_{0}^{2 / 3}(x)+b_{1} \rho_{0}(x)+b_{2} \rho_{0}^{4 / 3}(x) \\
& +b_{3} \rho_{0}^{5 / 3}(x)+\delta^{2}\left[b_{4} \rho_{0}(x)+b_{5} \rho_{0}^{4 / 3}(x)+b_{6} \rho_{0}^{5 / 3}\right],
\end{aligned}
$$

with $b_{1}=-741.28, b_{2}=1179.89, b_{3}=-467.54, b_{4}=148.26, b_{5}=372.84$, and $b_{6}=-769.57$. The $V_{0}(\mathrm{x})$ in Eq. 22 is the energy per particle of nuclear matter (in $\mathrm{MeV}$ ) which accounts for the neutron-proton asymmetry. $V_{C}$ is the coulomb energy of charge particle (proton) in a flucton,

$$
V_{C}=\frac{3}{5} \frac{Z^{2} e^{2}}{x}
$$

and $V_{C O}$ is the coulomb exchange energy given by

$$
V_{C O}=0.7386 Z e^{2}\left(3 Z / 4 \pi x^{3}\right)^{1 / 3} .
$$


On substituting $V_{0}(\mathrm{x})$ in Eq. 7 and taking its second order derivative, the symmetry energy $S_{0}^{N M}(\mathrm{x})$ of nuclear matter with density $\rho_{0}(\mathrm{x})$ is obtained:

$$
S_{0}^{N M}(x)=41.7 \rho_{0}^{2 / 3}(x)+b_{4} \rho_{0}(x)+b_{5} \rho_{0}^{4 / 3}(x)+b_{6} \rho_{0}^{5 / 3}(x) .
$$

The corresponding parameterized expressions for the pressure $P_{0}^{N M}(\mathrm{x})$ and the symmetry energy curvature $\Delta K_{0}^{N M}(\mathrm{x})$ for such a system within Brueckner energy density functional method have the forms

$$
P_{0}^{N M}(x)=27.8 \rho_{0}^{5 / 3}(x)+b_{4} \rho_{0}^{2}(x)+\frac{4}{3} b_{5} \rho_{0}^{7 / 3}(x)+\frac{5}{3} b_{6} \rho_{0}^{8 / 3}(x)
$$

and

$$
\Delta K_{0}^{N M}(x)=-83.4 \rho_{0}^{2 / 3}(x)+4 b_{5} \rho_{0}^{4 / 3}(x)+10 b_{6} \rho_{0}^{5 / 3}(x),
$$

respectively. These quantities are folded in the Eqs. (19-21) with the weight function to find the corresponding quantities of finite nuclei within the CDFM.

\section{Results and discussion}

\subsection{Ground state properties of the nuclei}

The main aim of this work is to study the effective surface properties like the symmetry energy $S$, neutron pressure $P$, and symmetry energy curvature $\Delta K$ for the isotopes (from neutron-deficient to the neutron-rich side of the nuclear landscape) of the light, heavy, and superheavy nuclei. Before proceeding to the effective surface properties, we have calculated their ground state bulk properties within the E-RMF model. Within E-RMF, we have used some of the recent force parameters like FSUGarnet [56], IOPB-I [57], and G3 [58]. The FSUGarnet [56], IOPB-I [57], and G3 [58] parameters have the advantage that their EoSs are softer compared to the NL3 parameter. The motive behind choosing these parameter sets has been illustrated in Ref. [55]. Table 1 contains the values of these parameter sets with the key EoS parameters for the nuclear matter at saturation (lower
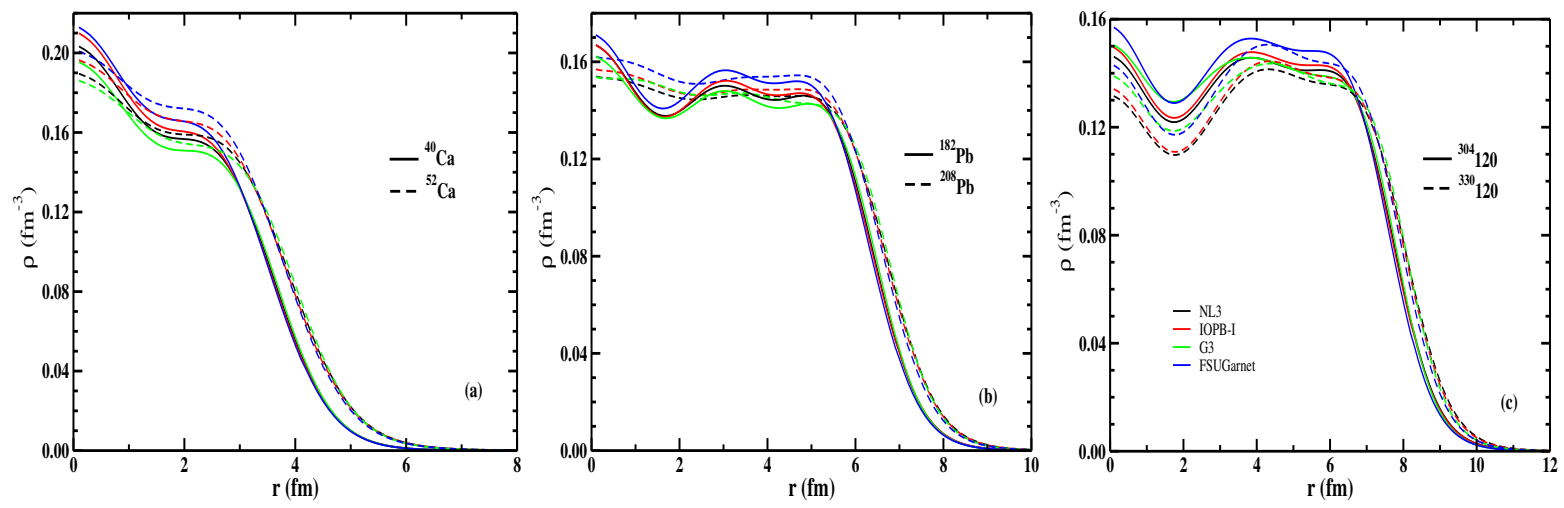

Figure 1. (color online) (a) The total density profiles for (a) ${ }^{40,52} \mathrm{Ca}$, (b) ${ }^{182,208} \mathrm{~Pb}$, and (c) ${ }^{304,330} 120$ as the representative cases corresponding to FSUGarnet [56], IOPB-I [57], G3 [58], and NL3 [59] parameter sets. 
Table 1. The FSUGarnet [56], IOPB-I [57], G3 [58], and NL3 [59] parameter sets are listed. The nucleon mass $M$ is $939.0 \mathrm{MeV}$ in all the sets. All the coupling constants are dimensionless, except $k_{3}$ which is in $\mathrm{fm}^{-1}$. The lower panel of the table shows the nuclear matter properties at saturation density $\rho_{0}\left(\mathrm{fm}^{-3}\right)$.

\begin{tabular}{|c|c|c|c|c|}
\hline & NL3 & FSUGarnet & G3 & IOPB-I \\
\hline$m_{s} / M$ & 0.541 & 0.529 & 0.559 & 0.533 \\
\hline$m_{\omega} / M$ & 0.833 & 0.833 & 0.832 & 0.833 \\
\hline$m_{\rho} / M$ & 0.812 & 0.812 & 0.820 & 0.812 \\
\hline$m_{\delta} / M$ & 0.0 & 0.0 & 1.043 & 0.0 \\
\hline$g_{s} / 4 \pi$ & 0.813 & 0.837 & 0.782 & 0.827 \\
\hline$g_{\omega} / 4 \pi$ & 1.024 & 1.091 & 0.923 & 1.062 \\
\hline$g_{\rho} / 4 \pi$ & 0.712 & 1.105 & 0.962 & 0.885 \\
\hline$g_{\delta} / 4 \pi$ & 0.0 & 0.0 & 0.160 & 0.0 \\
\hline$k_{3}$ & 1.465 & 1.368 & 2.606 & 1.496 \\
\hline$k_{4}$ & -5.688 & -1.397 & 1.694 & -2.932 \\
\hline$\zeta_{0}$ & 0.0 & 4.410 & 1.010 & 3.103 \\
\hline$\eta_{1}$ & 0.0 & 0.0 & 0.424 & 0.0 \\
\hline$\eta_{2}$ & 0.0 & 0.0 & 0.114 & 0.0 \\
\hline$\eta_{\rho}$ & 0.0 & 0.0 & 0.645 & 0.0 \\
\hline$\Lambda_{\omega}$ & 0.0 & 0.043 & 0.038 & 0.024 \\
\hline$\alpha_{1}$ & 0.0 & 0.0 & 2.000 & 0.0 \\
\hline$\alpha_{2}$ & 0.0 & 0.0 & -1.468 & 0.0 \\
\hline$f_{\omega} / 4$ & 0.0 & 0.0 & 0.220 & 0.0 \\
\hline$f_{\rho} / 4$ & 0.0 & 0.0 & 1.239 & 0.0 \\
\hline$f_{\rho} / 4$ & 0.0 & 0.0 & 1.239 & 0.0 \\
\hline$\beta_{\sigma}$ & 0.0 & 0.0 & -0.087 & 0.0 \\
\hline$\beta_{\omega}$ & 0.0 & 0.0 & -0.484 & 0.0 \\
\hline$\rho_{0}\left(\mathrm{fm}^{-3}\right)$ & 0.148 & 0.153 & 0.148 & 0.149 \\
\hline $\mathcal{E}_{0}(\mathrm{MeV})$ & -16.29 & -16.23 & -16.02 & -16.10 \\
\hline$M^{*} / M$ & 0.595 & 0.578 & 0.699 & 0.593 \\
\hline$J(\mathrm{MeV})$ & 37.43 & 30.95 & 31.84 & 33.30 \\
\hline$K_{\infty}(\mathrm{MeV})$ & 271.38 & 229.5 & 243.96 & 222.65 \\
\hline$L(\mathrm{MeV})$ & 118.65 & 51.04 & 49.31 & 63.58 \\
\hline$K_{\text {sym }}(\mathrm{MeV})$ & 101.34 & 59.36 & -106.07 & -37.09 \\
\hline$Q_{\text {sym }}(\mathrm{MeV})$ & 177.90 & 130.93 & 915.47 & 862.70 \\
\hline
\end{tabular}


Table 2. The calculated binding energy per particle (B/A), and charge radius $\left(R_{c h}\right)$ are compared with the available experimental data $[65,66]$. The predicted neutron skin-thickness $\Delta r=R_{n}-R_{p}$ is also depicted with all the four models and compared with the available experimental data [67].

\begin{tabular}{|c|c|c|c|c|c|c|}
\hline Nucleus & Obs. & Expt. & NL3 & FSUGarnet & G3 & IOPB-I \\
\hline \multirow[t]{3}{*}{${ }^{16} \mathrm{O}$} & $\mathrm{B} / \mathrm{A}$ & 7.976 & 7.917 & 7.876 & 8.037 & 7.977 \\
\hline & $\mathrm{R}_{c h}$ & 2.699 & 2.714 & 2.690 & 2.707 & 2.705 \\
\hline & $\mathrm{R}_{n}-\mathrm{R}_{p}$ & - & -0.026 & -0.029 & -0.028 & -0.027 \\
\hline \multirow[t]{3}{*}{${ }^{28} \mathrm{O}$} & $\mathrm{B} / \mathrm{A}$ & 5.988 & 6.379 & 5.933 & 6.215 & 6.220 \\
\hline & $\mathrm{R}_{c h}$ & - & 2.800 & 2.804 & 2.791 & 2.805 \\
\hline & $\mathrm{R}_{n}-\mathrm{R}_{p}$ & - & 0.809 & 0.796 & 0.741 & 0.809 \\
\hline \multirow[t]{3}{*}{${ }^{40} \mathrm{Ca}$} & $\mathrm{B} / \mathrm{A}$ & 8.551 & 8.540 & 8.528 & 8.561 & 8.577 \\
\hline & $\mathrm{R}_{c h}$ & 3.478 & 3.466 & 3.438 & 3.459 & 3.458 \\
\hline & $\mathrm{R}_{n}-\mathrm{R}_{p}$ & -0.08 & -0.046 & -0.051 & -0.049 & -0.049 \\
\hline \multirow[t]{3}{*}{${ }^{48} \mathrm{Ca}$} & $\mathrm{B} / \mathrm{A}$ & 8.666 & 8.636 & 8.609 & 8.671 & 8.638 \\
\hline & $\mathrm{R}_{c h}$ & 3.477 & 3.443 & 3.426 & 3.466 & 3.446 \\
\hline & $\mathrm{R}_{n}-\mathrm{R}_{p}$ & 0.16 & 0.229 & 0.169 & 0.174 & 0.202 \\
\hline \multirow[t]{3}{*}{${ }^{68} \mathrm{Ni}$} & $\mathrm{B} / \mathrm{A}$ & 8.682 & 8.698 & 8.692 & 8.690 & 8.707 \\
\hline & $\mathrm{R}_{c h}$ & - & 3.870 & 3.861 & 3.892 & 3.873 \\
\hline & $\mathrm{R}_{n}-\mathrm{R}_{p}$ & - & 0.262 & 0.184 & 0.190 & 0.223 \\
\hline \multirow[t]{3}{*}{${ }^{90} \mathrm{Zr}$} & $\mathrm{B} / \mathrm{A}$ & 8.709 & 8.695 & 8.693 & 8.699 & 8.691 \\
\hline & $\mathrm{R}_{c h}$ & 4.269 & 4.253 & 4.231 & 4.276 & 4.253 \\
\hline & $\mathrm{R}_{n}-\mathrm{R}_{p}$ & 0.09 & 0.115 & 0.065 & 0.068 & 0.091 \\
\hline \multirow[t]{3}{*}{${ }^{100} \mathrm{Sn}$} & $\mathrm{B} / \mathrm{A}$ & 8.253 & 8.301 & 8.298 & 8.266 & 8.284 \\
\hline & $\mathrm{R}_{c h}$ & - & 4.469 & 4.426 & 4.497 & 4.464 \\
\hline & $\mathrm{R}_{n}-\mathrm{R}_{p}$ & - & -0.073 & -0.078 & -0.079 & -0.077 \\
\hline \multirow[t]{3}{*}{${ }^{132} \mathrm{Sn}$} & $\mathrm{B} / \mathrm{A}$ & 8.355 & 8.371 & 8.372 & 8.359 & 8.352 \\
\hline & $\mathrm{R}_{c h}$ & 4.709 & 4.697 & 4.687 & 4.732 & 4.706 \\
\hline & $\mathrm{R}_{n}-\mathrm{R}_{p}$ & - & 0.349 & 0.224 & 0.243 & 0.287 \\
\hline \multirow[t]{3}{*}{${ }^{208} \mathrm{~Pb}$} & $\mathrm{~B} / \mathrm{A}$ & 7.867 & 7.885 & 7.902 & 7.863 & 7.870 \\
\hline & $\mathrm{R}_{c h}$ & 5.501 & 5.509 & 5.496 & 5.541 & 5.521 \\
\hline & $\mathrm{R}_{n}-\mathrm{R}_{p}$ & 0.17 & 0.283 & 0.162 & 0.180 & 0.221 \\
\hline
\end{tabular}


panel). From the table, we notice that the symmetry energy $(J)$ and its coefficients $L$, $K_{\text {sym }}$, and $\mathrm{Q}_{\text {sym }}$ are consistent with the allowed empirical and/or experimental ranges for three force parameters, namely FSUGarnet, G3, IOPB-I. The allowed empirical and/or experimental ranges along with the non-relativistic and relativistic constraints for a large number of force parameter sets can be found in Refs. [23, 24]. In case of NL3 force parameter, these values are overestimated to the allowed constraint ranges. These overestimations are due to the well-known stiffer nature of EoS. Furthermore, one can find that these ranges get broad with respect to density as well as higher order derivatives (i.e, the derived quantities from symmetry energy), which are the accepted behavior of mean field models. The binding energy per particle (B/A), charge radius $\left(R_{c h}\right)$, and neutron skin-thickness $\left(\Delta r=R_{n}-R_{p}\right)$ of some of the double magic nuclei for FSUGarnet [56], IOPB-I [57], G3, and NL3 [59] parameter sets are listed in Table 2
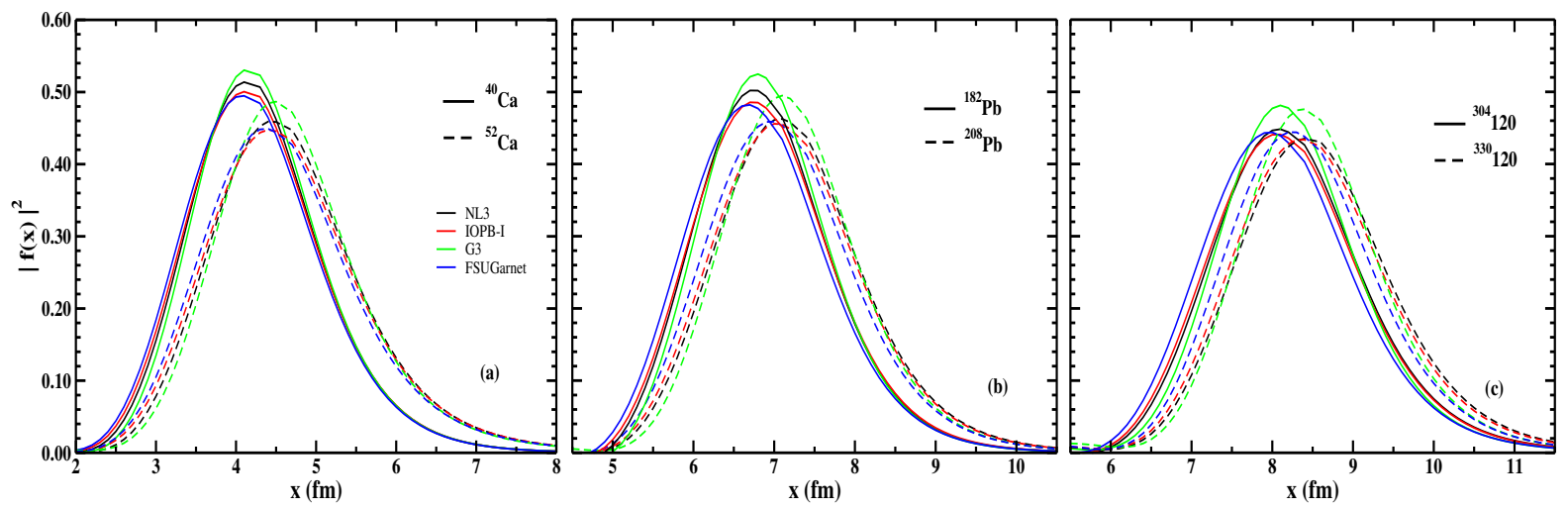

Figure 2. (color online) The weight function for (a) ${ }^{40,52} \mathrm{Ca}$, (b) ${ }^{182,208} \mathrm{~Pb}$, and (c) ${ }^{304,330} 120$ as the representative cases corresponding to FSUGarnet [56], IOPB-I [57], G3 [58], and NL3 [59] parameter sets.
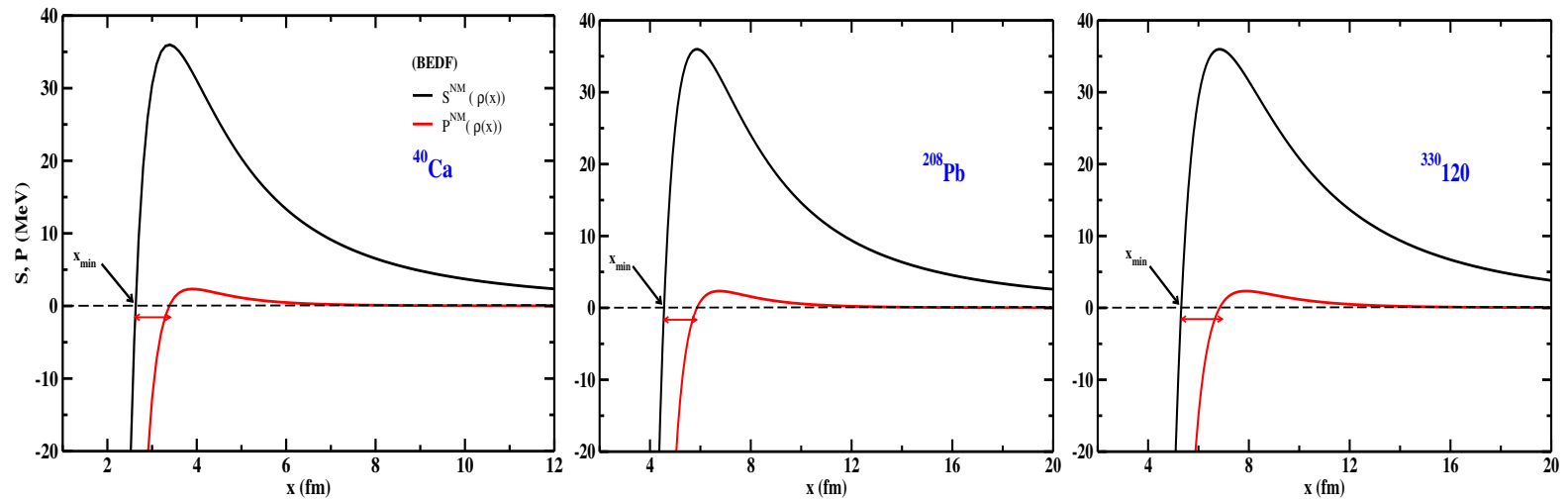

Figure 3. (color online) The symmetry energy $S^{N M}$, and the neutron presser $P^{N M}$ of nuclear matter at the Flucton's density of (a) $\mathrm{Ca}$, (b) $\mathrm{Pb}$, and (c) Z = 120 within the Brueckner energy density functional (BEDF) as a function of local coordinate $\mathrm{x}$. The $x_{\min }$ represent the lower limit of the integrations (Eqs. 19, 20, and 21). The negative values before the $x_{\min }$ points are the unphysical values. The dashed lines in the figure mark zero to differentiate between positive and negative values of $S^{N M}$, and $P^{N M}$. 
with the available experimental data $[65,66]$. The calculated properties of finite nuclei corresponding to all chosen parameter sets are in good agreement with each other. These results are comparable to the available experimental data. On inspecting the table, it is found that in some cases, the binding energy corresponding to the IOPB-I force parameter set overestimates the experimental data.

\subsection{Densities and weight functions for the nuclei}

It is important to note that the measurement of neutron density distribution in a nucleus is difficult task because of its neutral nature. As a result, the determination of neutron distribution radius $\mathrm{R}_{n}$ has been poorly done. On the other hand, the proton distribution radius $\mathrm{R}_{p}$ has been measured with a high accuracy. To solve this problem the recently proposed parity violation experiments PREX-II at JLab [34] and the Bates Laboratory at MIT were done with polarized beams and targets [68] which have given better results for the neutron distribution [34, 68]. The anti-proton experiment at CERN gives the neutron skin-thickness for 26 stable nuclei starting from ${ }^{40} \mathrm{Ca}$ to ${ }^{238} \mathrm{U}$ [67]. The recently reported G3 and IOPB-I force parameters in the framework of the E-RMF formalism reproduce the neutron skin-thickness $\Delta r$ quite well [57]. This gives us confidence that although the measurement of neutron distribution inside the nucleus is not as general as the proton even then our chosen forces should be capable enough to reproduce the proton and neutron distribution and hence the total density distribution of a nucleus.

The total densities of the nuclei, calculated within the spherically symmetric ERMF formalism corresponding to the NL3, IOPB-I, G3, and FSUGarnet parameter sets, are shown in Fig. 1. The color code is represented in the legends. The bold lines represent the total densities for neutron-deficient nuclei while dashed lines represent the densities for neutron-rich isotopes. The panels (a), (b), and (c) of the figure show the densities of ${ }^{40,52} \mathrm{Ca},{ }^{182,208} \mathrm{~Pb}$, and ${ }^{304,330} 120$, respectively, as the representative cases. It can be noticed from the figure that the central part of the density is larger for lighter
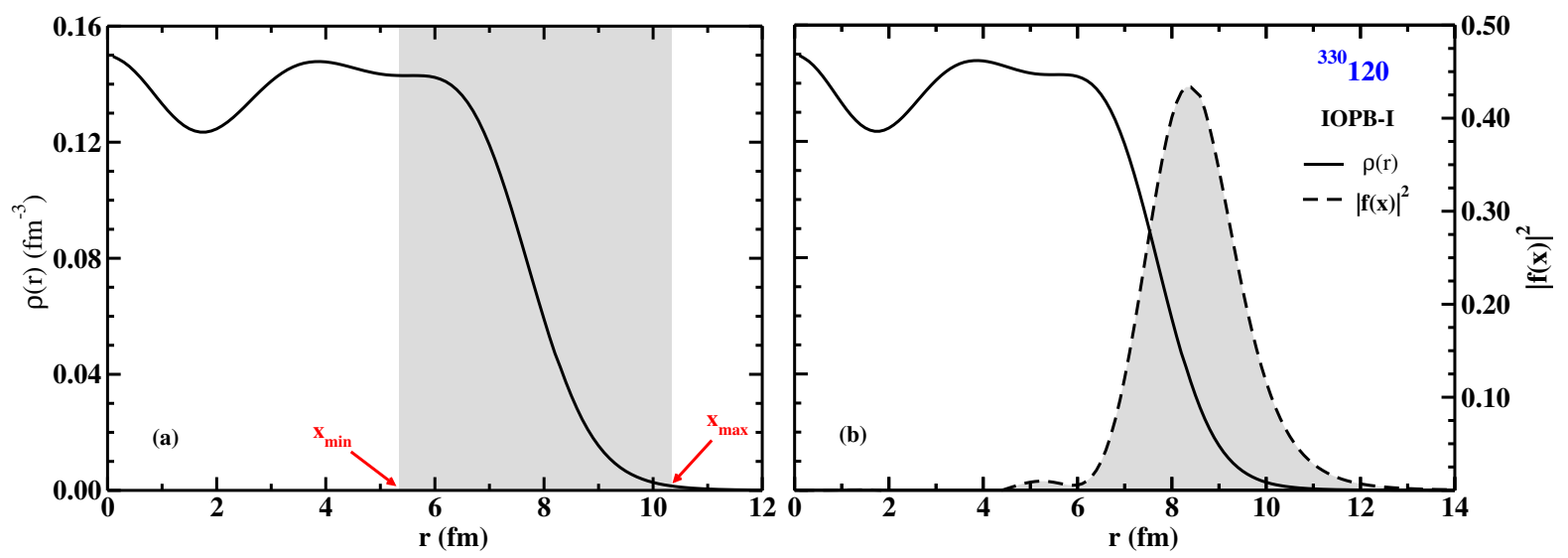

Figure 4. (color online) (a) The density for ${ }^{330} 120$ with the limits of integrations (Eqs. 19, 20, and 21) i.e., $x_{\min }$ and $x_{\max }$, (b) the weight function $|f(x)|^{2}(x=r)$ for ${ }^{330} 120$ along with its density, corresponding to IOPB-I parameter set. 
isotopes than those of heavier isotopes for particular nuclei. On the other hand, the surface densities are enhanced a bit as a function of radius for heavier isotopes than lighter.

The calculated densities from the E-RMF model are further used in Eq. 18 to obtain the weight functions for the corresponding nuclei. The weight functions for the nuclei ${ }^{40,52} \mathrm{Ca},{ }^{182,208} \mathrm{~Pb}$, and ${ }^{304,330} 120$ as the representative cases are shown in the panels (a), (b), and (c) of Fig. 2, respectively. From the figure, one can notice that the trend of the density profile (Fig. 1) is reflected in weight functions. In other words, the lower value of the central density gives the lesser height of the weight function for an isotope of the particular nucleus. Further, it can be noticed in the figure that the maxima of the weight functions shift towards the right (larger $r$ ) with the size of a nucleus increases. The G3 parameter set predicts the larger weight function for all the nuclei while the lower one corresponds to the FSUGarnet parameter set. The symmetry energy, neutron pressure and symmetry energy curvature of nuclear matter at local coordinate are folded with the calculated weight function of a nucleus which results in the corresponding effective surface properties of the finite nucleus. It would be worth illustrating the point that why these quantities are termed as the surface properties and how to find the limits of integration (Eqs. 19-21).

In principle, the limits of integration in Eqs. $19-21$ are set from 0 to $\infty$. But, the symmetry energy of infinite symmetric nuclear matter within the Brueckner energy density functional method has some negative values (unphysical points) in certain regions. In order to avoid the unphysical points of the symmetry energy of nuclear matter, the limits of integration $x_{\min }$ and $x_{\max }$ are put other than what mentioned above. In general, $x_{\min }$ and $x_{\max }$ are the points where the symmetry energy of nuclear matter changes from negative to positive and from positive to negative, respectively [49]. For the better understanding of the concept of finding $x_{\min }$ and $x_{\max }$, we present the symmetry energy of nuclear matter within Brueckner energy density functional $S^{N M}(x)$ (used in Eq. 19) for ${ }^{40} \mathrm{Ca},{ }^{208} \mathrm{~Pb}$, and ${ }^{330} 120$ in the panels (a), (b), and (c) of Fig. 3. The nature of the curves for the symmetry energy of nuclear matter is the same for the nuclei shown in the figure with almost the same maximum value. However, the curves shift towards the right (larger values of $r$ ) with nuclei having a large mass number. It can easily be noticed from the figure that at $x=2.5 \mathrm{fm}, x=4.3 \mathrm{fm}$, and $x=5.2 \mathrm{fm}$, the $S^{N M}(x)$ of nuclear matter changes from negative to positive at Flucton density in ${ }^{40} \mathrm{Ca},{ }^{208} \mathrm{~Pb}$, and ${ }^{330} 120$, respectively. Thus, these points are considered as $x_{\min }$ for the respective nuclei. While no point at large $x$ seems to be such that where the $S^{N M}(x)$ changes from positive to negative (in this case). Rather, $S^{N M}(x)$ tends to zero at large values of $x$. Thus, the value of $x_{\max }$ can not be fixed in this way. On the other hand, the densities of the nuclei become almost zero at $r=6.0 \mathrm{fm}, r=9.4 \mathrm{fm}, r=10.5 \mathrm{fm}$ for ${ }^{40} \mathrm{Ca}$, ${ }^{208} \mathrm{~Pb}$, and ${ }^{330} 120$, respectively. Therefore, these points are considered as the $x_{\max }$ (upper limit of integrations (Eqs. 19 - 21)). Figure 4 represents the density of ${ }^{330} 120$ with the IOPB-I parameter set as the representative case, showing the limits of integration $x_{\min }$ and $x_{\max }$. The limits are used in Eqs. (19-21) to find the symmetry energy, neutron 
pressure, and symmetry energy curvature, respectively. It can be noticed from Fig. 4 that the values of limit do not have any central part of the density and lie in the surface region. Hence, the quantities $\mathrm{S}, \mathrm{P}$, and $\Delta K$ are known as surface properties. For further illustrating the concept of referring these quantities as the surface properties, the panel (b) of Figure 4 is presented, here, showing the density and weight function altogether. It has already been mentioned that the properties of infinite nuclear matter are folded with the weight function to obtain the corresponding quantities of finite nuclei. The significant values of weight functions (its peak value) lie in the range which corresponds to the surface part of the density. This is also one of the reasons to call these quantities as the surface properties.

\subsection{The effective surface properties of the nuclei}

Before discussing the results of the symmetry energy and its derivatives, it is important to brief why we have considered spherical densities of the isotopes despite the fact that some of the isotopes, considered here, might be deformed. Generally, the symmetry energy coefficient of a finite nucleus is a bulk property, which mainly depends on the isospin asymmetry of the nucleus. Further, the symmetry energy coefficient of a nucleus can be written in terms of the volume and surface-symmetry energy coefficients (see Eq. 2 of Ref. [69]). It is remarked in [70] that the volume-symmetry energy is shapeindependent, so the surface effects do not play their role in the volume-symmetry energy. On the other hand, the surface effects become negligible for heavy and superheavy nuclei since the surface symmetry energy coefficient is proportional to $A^{-1 / 3}$, where $A$ is the mass number $[69,70]$. It is clear from the previous sentence that surface effects are important for lighter nuclei and have negligible effects for heavier nuclei. The inclusion of the deformation may slightly improve the results but the effects of deformation are very small. In some of the recent works [71], authors have shown the effect of deformation on the symmetry energy of finite nuclei using Thomas-Fermi approximation over Skyrme energy density functional. Following the work, one can find the relative change in the symmetry energy with very large deformation $\left(\beta_{2} \sim 0.6\right)$ is around $0.4 \mathrm{MeV}$. It is mentioned therein [71] that the effects of deformation decrease with respect to the mass number. So for the sake of computational ease, we have considered the spherical densities of the isotopes.

The effective surface properties for the isotopic series of $\mathrm{O}, \mathrm{Ca}, \mathrm{Ni}, \mathrm{Zr}, \mathrm{Sn}$, and $\mathrm{Pb}$ nuclei are shown in Fig. 5. The first, second, and third row of each panel of the figure represent the symmetry energy $S$, neutron pressure $P$, and symmetry energy curvature $\Delta K$, respectively. The value of the symmetry energy for finite nuclei lie in the range of 24-31 MeV. It is observed from the figure that the symmetry energy is larger for the FSUGarnet parameter set for all the cases except the isotopes of $\mathrm{O}$ and few isotopes of Ca. While G3 set predicts less symmetry energy in most of the cases in Fig. 5. The nature of parameter sets get reversed in the cases of neutron pressure $P$ and symmetry energy curvature $\Delta K$. For example, the G3 parameter set predicts the larger value of $P$ 
and $\Delta K$ for all the isotopic series except $N=138$ isotope of $\mathrm{Pb}$. Furthermore, we find several peaks at neutron numbers, which correspond to the magic numbers and/or shell /sub-shell closures for each isotopic chain. These peaks in the symmetry energy curve imply that the stability of the nuclei at the magic neutron number is more as compared to the neighboring isotopes. The peaks in the symmetry energy curve imply that more energy would be required to convert one neutron to proton or vice verse. Apart from the peaks of the symmetry energy at the magic neutron number, a few small peaks are
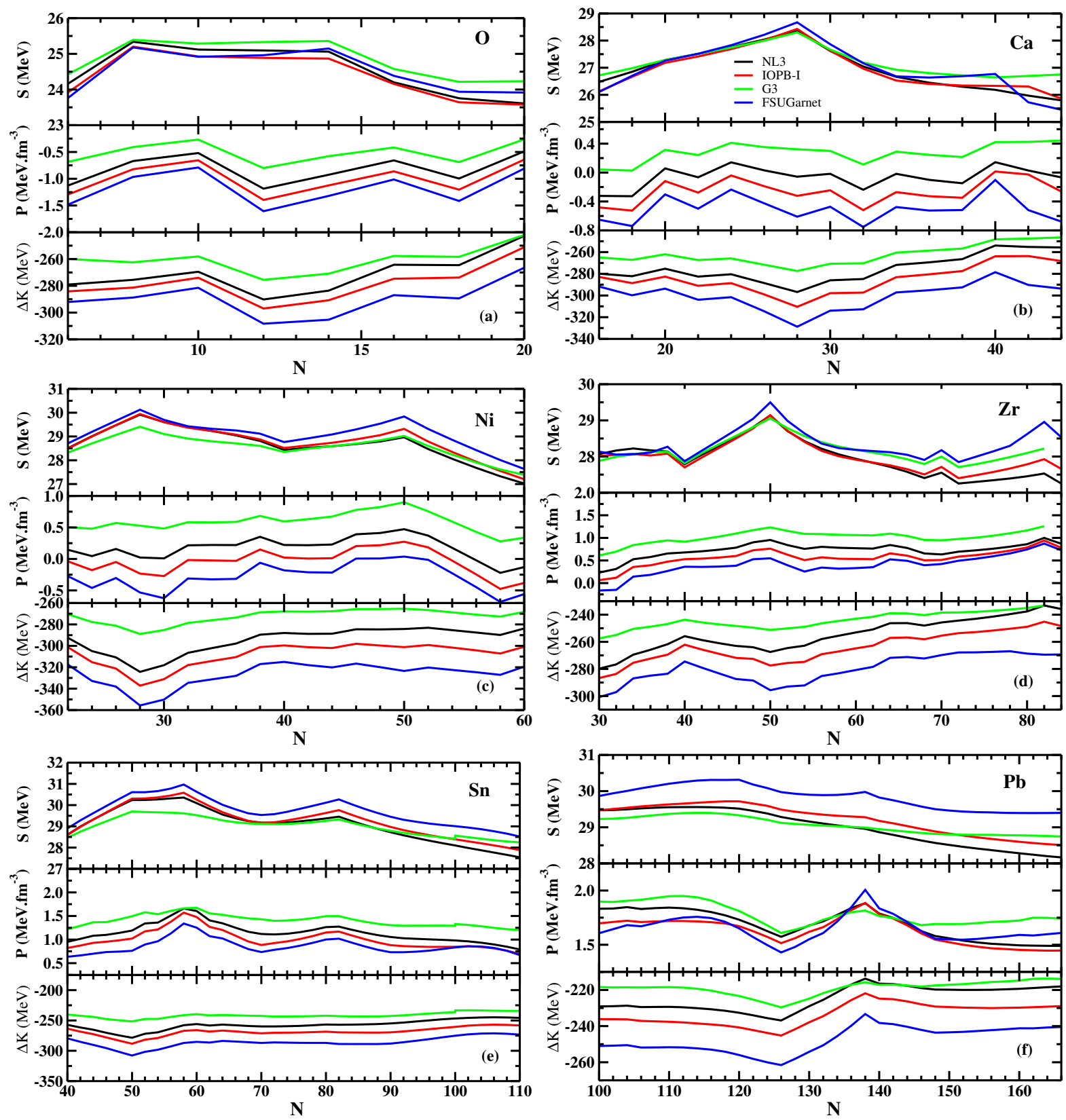

Figure 5. (color online) The symmetry energy $(S)$, pressure $(P)$, and symmetry energy curvature $(\Delta K)$ for the isotopic series of $\mathrm{O}, \mathrm{Ca}, \mathrm{Ni}, \mathrm{Zr}, \mathrm{Sn}$, and $\mathrm{Pb}$ nuclei corresponding to NL3, IOPB-I, G3, and FSUGarnet parameters sets. 
also evolved which may arise due to the shell structure on the density distribution of the nuclei. The present investigation predicts a few neutron magic numbers beyond the known magic numbers based on the well-known feature of symmetry energy over an isotopic chain, which will be studied systematically in the near future.

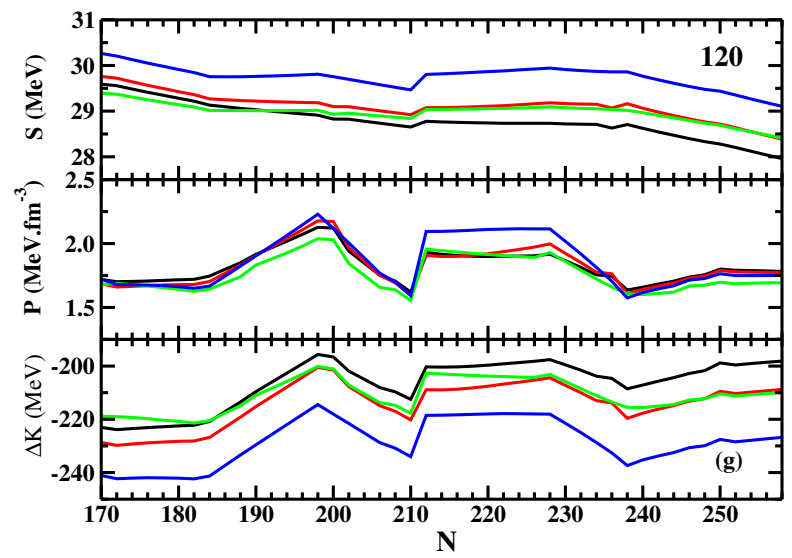

Figure 6. (color online) The description is same as in Fig. 5 but for $\mathrm{Z}=120$ nuclei.
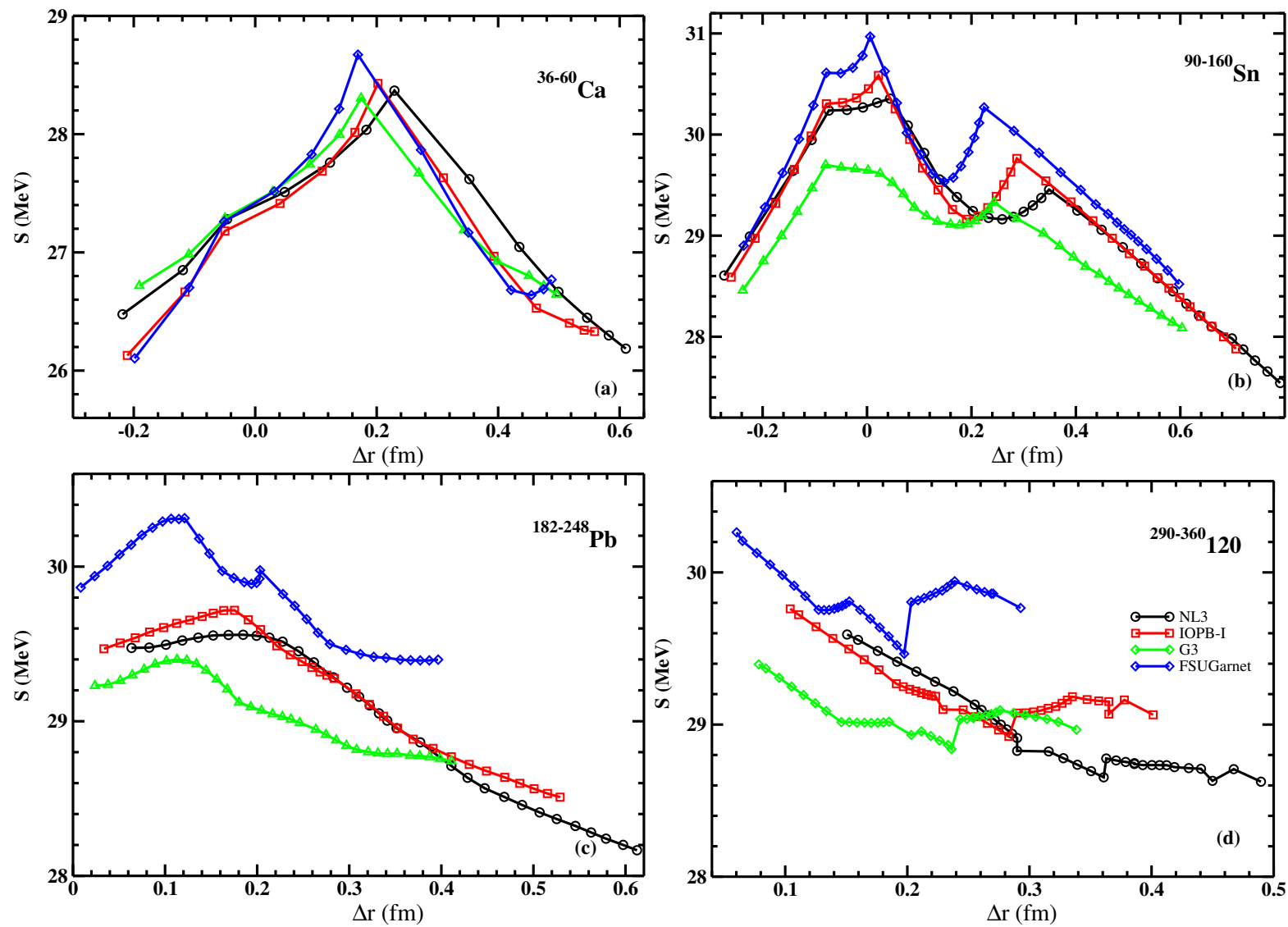

Figure 7. (color online) The correlation of the neutron skin-thickness with the symmetry energy is shown for the isotopes of $\mathrm{Ca}, \mathrm{Sn}, \mathrm{Pb}$, and $\mathrm{Z}=120$ nuclei as the representative cases corresponding to NL3, IOPB-I, G3, and FSUGarnet parameter sets. 
The neutron pressure $P$ and symmetry energy curvature $\Delta K$ have the opposite nature to that of the symmetry energy with respect to the force parameter sets. It is meant by the opposite nature that higher the symmetry energy of nuclei corresponding to the particular interaction, lower the neutron pressure and symmetry energy curvature values are for the same parameter set and vice verse. Further, we found negative neutron pressure for the isotopes of Oxygen nuclei for all parameter sets. Also negative values of $P$ are obtained for the isotopic series of $\mathrm{Ca}$ and Ni corresponding to the FSUGarnet parameter set and a few of them corresponding to IOPB-I set. However, the NL3 set predicts negative values of $P$ for some of the isotopes of $\mathrm{Ca}$ and neutron-rich isotopes of $\mathrm{Ni}$. It is to note that the negative value of $P$ arises due to the significant value of weight function (reflection of the behaviour of density distribution) in the range of local coordinate $\mathrm{x}(\mathrm{fm})$, where the pressure of nuclear matter is negative. For example, in Fig. 3 (a), the red arrow bar represents the range of $x$ wherein the pressure is negative. In this range of $\mathrm{x}$, the weight function has non-zero definite value (see Fig. 2 (a)), which is when multiplied by the pressure of nuclear matter (in Eq. 20), results in the negative pressure of a nucleus. The non-zero definite values of the weight functions in the lower range of $\mathrm{x}$ are obtained for lighter nuclei due to their small size. On the other hand, the weight functions have negligible values for heavier nuclei in the range of $\mathrm{x}$ wherein the pressure of nuclear matter is negative for the corresponding nuclei. In general, the pressure and symmetry energy curvature values increase with neutron number for an isotopic series while the symmetry energy decreases with the increase of neutron number.

Observing the behavior of effective symmetry energy, neutron pressure, and symmetry energy curvature, we find a kink and/or a fall at the magic (or shell closures) neutron numbers. In the case of the isotopic chain of $\mathrm{Pb}$ nucleus, we did not get a kink in the $S$ curve at $\mathrm{N}=126$. In spite of that, there is a fall of the $S$ curve at $\mathrm{N}=126$, beyond which it is almost constant for the few isotopes. This signifies that $\mathrm{N}=126$ as a weak magic number. Moreover at $\mathrm{N}=126$, the sharp fall of the $P$ and $\Delta K$ curves can be noticed from the figure as they are related with the first and second-order derivatives of the symmetry energy. Even the sharp kink is not observed in the $S$ curve at N=126 for the isotopic series of $\mathrm{Pb}$ likewise at the other neutron magic numbers in different isotopic series, the sharp fall in the $P$ and $\Delta K$ curves support $\mathrm{N}=126$ as a magic number. Furthermore, in Fig. 5 of Ref. [26], one can find that there are small kinks/deviation in the $S$ and $P$ curves at ${ }^{208} \mathrm{~Pb}$ while sharp kinks are observed at the other neutron magic numbers in the isotopic series of $\mathrm{Ni}$ (Fig. 1) and Sn (Fig. 6) [26].

The effective surface properties for the isotopic series of $\mathrm{O}, \mathrm{Ca}, \mathrm{Ni}, \mathrm{Zr}, \mathrm{Sn}, \mathrm{Pb}$ nuclei motivate us to pursue the said calculations for isotopes of experimentally unknown superheavy nuclei. Recently, the analysis of the superheavy element is a frontier topic in nuclear physics. The discovery of transuranic elements from $Z=93-118$ with Oganesson $\left({ }_{118} \mathrm{Og}\right)$ is the heaviest element known so far that completes the $7 p$ orbitals. Hence, the next element $\mathrm{Z}=119$ will occupy a new row in the Periodic Table. A large number of models predict different neutron and proton combinations for the next double close 
magic nuclei in the superheavy stability valley [72]. Among them, $Z=120$ attracts much attention with neutron number $\mathrm{N}=184$ as the next double magic isotope, and near to be synthesized. Therefore, we have calculated the effective surface properties for the isotopic chain of $\mathrm{Z}=120$ nuclei, shown in Fig. 6. We found almost a smooth fall in the symmetry energy up to $\mathrm{N}=210$, and further, a very miniature growth appears up to $\mathrm{N}=240$ following the previous trends. In other words, there is a moderate decrease in the symmetry energy over the isotopic chain of $\mathrm{Z}=120$ with some exception for the neutron number $212 \leq \mathrm{N} \leq 238$ (see Fig. 6). Here, we also got peaks in the neutron-rich side at $\mathrm{N}=212$ and 238 in the symmetry energy curve as similar to those in Fig. 5. These neutron numbers can be attributed to the magic neutron numbers. Here also, the symmetry energy predicted by the FSUGarnet parameter set is larger compared to the rest of the parameter sets. The neutron pressure and symmetry energy curvature are shown, respectively, in the second and third rows of Fig. 6. Similar conclusions can be drawn for the $P$ and $\Delta K$ of $\mathrm{Z}=120$ isotopes, as for other isotopic chains. In general, we get a bit larger values of the effective surface properties for $Z=120$ isotopes as compared to the isotopes of the rest of the nuclei except a few isotopes of $\mathrm{Sn}$ and $\mathrm{Pb}$. Here, we did not find any transparent signature of shell closures or magicity in the symmetry energy over the isotopic chain of $\mathrm{Z}=120$. Following Ref. [73], the ground state configuration of $\mathrm{Z}=120$ isotopes are super-deformed prolate and/or oblate shapes followed by a spherical intrinsic excited state. Here, our calculation limited to the spherical co-ordinate, which may cause for weaken the signature over the isotopic chain. Hence, a self-consistent microscopic calculation is required in the deformed basis.

\subsection{Correlation of skin-thickness with the symmetry energy}

The skin-thickness is correlated with the surface properties [26, 35, 48, 49] for a different series of isotopic nuclei. It was found to be linearly correlated with the surface properties except for some kinks, which correspond to the magic/semi-magic nuclei of an isotopic chain $[26,35,48,49]$. Here, we present the correlation between the symmetry energy and the neutron skin-thickness for the isotopic series of $\mathrm{Ca}, \mathrm{Sn}, \mathrm{Pb}$, and Z $=120$ nuclei for the NL3, IOPB-I, G3, and FSUGarnet parameter sets. It is remarked in Refs. [55, 57] and shown in Table 2 that stiffer EoS of nuclear matter predicts the larger neutron skinthickness of nuclei. Among the chosen parameter sets, NL3 is the stiffest which predicts larger skin-thickness, while FSUGarnet as being softer estimate smaller skin-thickness. On the other hand, it has been shown in Fig. 5 that the symmetry energy is maximum at the neutron magic number of an isotope.

Fig. 7 shows the correlation of the symmetry energy with the neutron skin-thickness of nuclei. The panels (a), (b), (c), and (d) of the figure represent the correlation for the isotopic series of $\mathrm{Ca}, \mathrm{Sn}, \mathrm{Pb}$, and $\mathrm{Z}=120$, respectively. It is clear from the figure that the skin-thickness of the nuclei are larger corresponding to the NL3 parameter set and

smaller for FSUGarnet set. It can be noticed from the figure that with some exceptions the symmetry energy predicted by FSUGarnet is higher compared to the rest of the 
parameter sets. The peaks in the symmetry energy curves (in Fig. 7) correspond to the magic or semi-magic neutron numbers. The symmetry energy decreases with varying neutron numbers in either direction of the magic/semi-magic number. It implies that for exotic nuclei (nuclei lie at the drip-line) less amount of energy is required to convert one proton to neutron or vice-versa, depending on the neutron-proton asymmetry. The behavior of the symmetry energy with skin-thickness is undermined for a few cases. For the cases of $\mathrm{Ca}, \mathrm{Sn}$, and $\mathrm{Pb}$, the symmetry energy curve is almost linear before and after the peaks. Further improvement in the results can be obtained by solving the field equations in an axially deformed basis.

\section{Summary and Conclusions}

In summary, we have studied the effective surface properties like the symmetry energy, neutron pressure, and the symmetry energy curvature for the isotopic series of $\mathrm{O}$, Ca, $\mathrm{Ni}, \mathrm{Zr}, \mathrm{Sn}, \mathrm{Pb}$, and Z $=120$ nuclei within the coherent density fluctuation model. We have used the spherically symmetric effective field theory motivated relativistic mean field model to study the ground state bulk properties of nuclei with the recent parameter sets like IOPB-I, FSUGarnet, and G3. The calculated results are compared with the predictions by the well known NL3 parameter set and found in good agreement. The densities of nuclei calculated within the E-RMF formalism are used as the inputs to the coherent density fluctuation model to obtain the weight functions for the isotopes. The symmetry energy, neutron pressure, and symmetry energy curvature of infinite nuclear matter are calculated within Brueckner energy density functional model which are further folded with the weight function to find the corresponding quantities of finite nuclei. The FSUGarnet parameter set predicts the large value of the symmetry energy while the smaller symmetry energy values are for G3 set with some exceptions. We found a larger value of the skin-thickness for the force parameter that corresponds to the stiffer EoS and vice-versa. We also found a few mass-dependence peaks in the symmetry energy curve corresponding to the neutron magic/semi-magic number. Observing the nature of the symmetry energy over the isotopic chain, we predict a few neutron magic numbers in the neutron-rich exotic nuclei including superheavy. The transparent signature of magicity is diluted for a few cases over the isotopic chain of $\mathrm{Pb}$ and $\mathrm{Z}=$ 120 nuclei. Similar behavior is also observed for the neutron pressure and symmetry energy curvature for these isotopes. Concurrently, the present calculations tentatively reveal a way to calculate the effective surface properties of unknown drip-line nuclei including superheavy. More detail studies are disclosed by considering deformation into account. The calculated quantities are important for the structural properties of finite nuclei and may be useful for the synthesis of neutron-rich or superheavy nuclei. These effective surface properties can also be used to constrain an EoS of the nuclear matter and consequently nucleosynthesis processes.

Acknowledgments: We are thankful to Shakeb Ahmad and Mitko Gaidarov for

the fruitful discussions. AQ would like to acknowledge the Department of Science and 
Technology (DST), Gov. of India for providing financial support in the form of INSPIRE fellowship with No. DST/INSPIRE fellowship/2016/IF160131. The author (MB) has been supported by FAPESP Project Nos. 2014/26195-5 and 2017/05660-0, INCT-FNA Project Nos. 464898/2014-5 and by the CNPq -Brasil.

\section{References}

[1] Yu. Ts. Oganessian at al, 2006 Phys. Rev. C 74, 044602.

[2] M. S. Mehta, H. Kaur, Bharat Kumar, and S. K. Patra, 2015 Phys. Rev. C 92, 054305.

[3] Elena Litvinova, 2012 Phys. Rev. C 85, 021303(R).

[4] W. -T. Chou, R. F. Casten and N. V. Zamfir, 1995 Phys. Rev. C 51, 2444.

[5] T. Otsuka, R. Fujimoto, Y. Utsuno, B. A. Brown, M. Honma, and T. Mizusaki, 2001 Phys. Rev. Lett. 87, 082502.

[6] M. Leino, J. Ãystõ, T. Enqvist, P. Heikkinem, A. Jokinen, M. Nurmia, A. Ostrowski, W. H. Trazaska, J. Uusitalo, K. Eskola, P. Armbruster, and V. Ninov, 1995 Nucl. Instrum. Methods Phys. Res. B 99, 653.

[7] M. Thoennessen, 2010 Nucl. Phys. A 834, 688c.

[8] H. Geissel, P. Armbruster, K. H. Behr, A. Brũnle, K. Burkard, M. Chen, H. Folger, B. Franczak, H. Keller, O. Klepper, B. Langenbeck, F. Nickel et al., 1992 Nucl. Instrum. Methods Phys. Res. $B$ 70, 286.

[9] M. Winkler, H. Geissel, H. Weick, B. Achenbach, K.-H. Behr, D. Boutin, A. Brü, M. Gleim, W. Hũller, C. Karagiannis, A. Kelic, B. Kindler et al., 2008 Nucl. Instrum. Methods Phys. Res. B 266, 4183.

[10] A. C. Müller and R. Anne, 1991 Nucl. Inst. Methods Phys. Res. B 56, 559.

[11] A. M. Rodin, S. V. Stepantsov, D. D. Bogdanov, M. S. Golovkov, A. S. Fomichev, S. I. Sidorchuk, R. S. Slepnev, R. Wolski, G. M. Ter-Akopian, Y. T. Oganessian, A. A. Yukhimchuk, V. V. Perevozchikov et al., 2003 Nucl. Instrum. Methods Phys. Res. B 204, 114.

[12] Z. Sun, W. L. Zhan, Z. Y. Guo, G. Xiao, and J. X. Li, 2003 Nucl. Instrum. Methods Phys. Res. A 503, 496.

[13] M. Winkler, H. Geissel, H. Weick, B. Achenbach, K.-H. Behr, D. Boutin, A. Brü, M. Gleim, W. Hũller, C. Karagiannis, A. Kelic, B. Kindler et al., 2008 Nucl. Instrum. Methods Phys. Res. B 266, 4183.

[14] C. J. Gross, T. N. Ginter, D. Shapira, W. T. Milner, J. W. McConnell, A. N. James, J. W. Johnson, J. Mas, P. F. Mantica, R. L. Auble, J. J. Das, J. L. Blankenship et al., 2000 Nucl. Instrum. Methods Phys. Res. A 450, 12.

[15] T. Nikŝić, D. Vretenar, and P. Ring, 2008 Phys. Rev. C 78, 034318.

[16] N. Van Giai, B. V. Carlson, Z. Ma, and H. Wolter, 2010 J. Phys. G 37, 064043.

[17] E. N. E. van Dalen and H. Müther, 2010 Int. J. Mod. Phys. 19, 2077.

[18] V. Rodin, 2007 Prog. Part. Nucl. Phys. 59, 268.

[19] B.-A. Li, L.-W. Chen, and C. M. Ko, 2008 Phys. Rep. 464, 113.

[20] M. Colonna, 2009 J. Phys. Conf. Ser. 168, 012006.

[21] A. W. Steiner, M. Prakash, J. M. Lattimer, and P. J. Ellis, 2005 Phys. Rep. 411, 325.

[22] F. J. Fattoyev, W. G. Newton, J. Xu and B.-A. Li, 2012 Phys. Rev. C 86, 025804.

[23] M. Dutra, O. Lourenço, J. S. Sá Martins, A. Delfino, J. R. Stone, and P. D. Stevenson, 2012 Phy. Rev. C 85, 035201.

[24] M. Dutra, O. Lourenço, S. S. Avancini, B. V. Carlson, A. Delfino, D. P. Menezes, C. Providència, S. Typel, and J. R. Stone, 2014 Phys. Rev. C 90, 055203.

[25] Nuclear Astrophysics, in The 2015 Long Range Plan for Nuclear Science, https : //science.energy.gov/ /media/np/nsac/pdf/2015LRP/2015 ${ }_{L} R P N S_{0} 91815 . p d f \quad$ (USDOE, 2015), p. 53. 
[26] M. K. Gaidarov, A. N. Antonov, P. Sarriguren, and E. Moya de Guerra, 2011 Phys. Rev. C 84, 034316.

[27] W. D Myers and J. Swiatecki, 1980 Nucl. Phys. A 336, 267.

[28] P. Danielewicz, 2003 Nucl. Phys. A 727, 233.

[29] J. M. Lattimer and M. Prakash, 2007 Phys. Rept. 442, 109.

[30] B. A. Brown, 2000 Phys. Rev. Lett. 85, 5296.

[31] R. J. Furnstahl, 2002 Nucl. Phys. A 706, 85.

[32] M. Centelles, X. Roca-Maza, X. Viñas, and M. Warda, 2009 Phys. Rev. Lett. 102, 122502.

[33] X. Roca-Maza, M. Centelles, X. Viñas, and M. Warda, 2011 Phys. Rev. Lett. 106, 252501.

[34] S. Abrahamyan et al., (PREX Collaboration) 2012 Phys. Rev. Lett. 108, 112502.

[35] M. Bhuyan, B. V. Carlson, S. K. Patra, and S.-G. Zhou, 2018 Phys. Rev. C 97, 024322.

[36] J. M. Lattimer, 2014 Nucl. Phys. A 928, 276.

[37] Abdul Quddus, M. Bhuyan, Shakeb Ahmad, B. V. Carlson, and S. K. Patra, 2019 Phys. Rev. C 99, 044314 .

[38] W. D. Myers and W. J. Swiatecki, 1966 Nucl. Phys. A 81, 1.

[39] P. Möller et al., 1995 At. Data Nucl. Data Tables 59, 185.

[40] K. Pomorski and J. Dudek, 2003 Phys. Rev. C 67, 044316.

[41] L.-W. Chen, C. M. Ko, and B.-A. Li, 2005 Phys. Rev. C 72, 064309.

[42] S. Yoshida and H. Sagawa, 2006 Phys. Rev. C 73, 044320.

[43] L.-W. Chen, C. M. Ko, B.-A. Li, and J. Xu, 2010 Phys. Rev. C 82, 024321.

[44] A. Carbone, G. Coló, A. Bracco, L. -G. Cao, P. F. Bortignon, F. Camera, and O. Wieland, 2010 Phys. Rev. C 81, 041301(R).

[45] C.-H. Lee, T. T. S. Kuo, G. Q. Li, and G. E. Brown, 1998 Phys. Rev. C 57, 3488.

[46] B. K. Agrawal, 2010 Phys. Rev. C 81, 034323.

[47] D. Vretenar, T. Nikŝić, and P. Ring, 2003 Phys. Rev. C 68, 024310.

[48] A. N. Antonov, V. A. Nikolaev, and I. Zh. Petkov, 1979 Bulg. J. Phys. 6, 151; 1980 Z. Phys. A 297, 257; 1982 304, 239; 1985 Nuovo Cimento A 86, 23; A. N. Antonov et al., ibid. 1989 102, 1701; A. N. Antonov, D. N. Kadrev, and P. E. Hodgson, 1994 Phys. Rev. C 50, 164.

[49] M. K. Gaidarov, A. N. Antonov, P. Sarriguren, and E. M. de Guerra, 2012 Phys. Rev. C 85, 064319.

[50] K. A. Brueckner, J. R. Buchler, S. Jorna, and R. J. Lombard, 1968 Phys. Rev. 171, 1188.

[51] K. A. Brueckner, J. R. Buchler, R. C. Clark, and R. J. Lombard, 1969 Phys. Rev. 181, 1543.

[52] A. N. Antonov, P. E. Hodgson, and I. Zh. Petkov, Nucleon Momentum and Density Distributions in Nuclei (Clarendon Press, Oxford, 1988); Nucleon Correlations in Nuclei (Springer, Berlin, 1993).

[53] R. J. Furnstahl, B. D. Serot and H. B. Tang, 1996 Nucl. Phys. A 598, 539; R. J. Furnstahl, B. D. Serot and H. B. Tang, 1997 Nucl. Phys. A 615, 441.

[54] M. Del Estal, M. Centelles, X. Viñas and S. K. Patra, 2001 Phys. Rev. C 63, 044321; M. Del Estal, M. Centelles, X. Viñas and S.K. Patra, 2001 Phys. Rev. C 63, 024314.

[55] Abdul Quddus, K. C. Naik, and S. K. Patra, 2018 J. Phys. G: Nucl. Part. Phys. 45, 075102.

[56] Wei-Chai Chen and J. Piekarewicz, 2015 Phys. Lett. B 748, 284.

[57] Bharat Kumar, S. K. Patra, and B. K. Agrawal, 2018 Phys. Rev. C 97, 045806.

[58] Bharat Kumar, S. K. Singh, B. K. Agrawal, S. K. Patra, 2017 Nucl. Phys. A 966, 197.

[59] G. A. Lalazissis, J. König and P. Ring, 1997 Phys. Rev. C 55, 540.

[60] D. Vautherin and D. M. Brink, 1972 Phys. Rev. C 5, 626.

[61] E. Chabanat, P. Bonche, P. Haensel, J. Meyer, and R. Schaeffer, 1998 Nucl. Phys. A635, 231.

[62] A. N. Antonov, D. N. Kadrev, and P. E. Hodgson, 1994 Phys. Rev. C 50, 164.

[63] C. Fuchs, H. Lenske, and H. H. Wolter, 1995 Phys. Rev. C 52, 3043.

[64] A. N. Antonov, D. N. Kadrev, M. K. Gaidarov, P. Sarriguren, and E. Moya de Guerra, 2017 Phys. Rev. C 95, 024314.

[65] M. Wang, G. Audi, A. H. Wapstra, F. G. Kondev, M. MacCormick, X. Xu and B. Pfeiffer, 2012 
Chin. Phys. C 36, 1603.

[66] I. Angeli, K. P. Marinova, 2013 At. Data and Nucl. Data Tables 99, 69.

[67] A. Trzcińska, J. Jastrzebski, P. Lubiński, F. J. Hartmann, R. Schmidt, T. von Egidy, and B. Klos, 2001 Phys. Rev. Lett. 87, 082501; J. Jastrzebski, A. Trzcińska, P. Lubiński, B. Klos, F. J. Hartmann, T. von Egidy, and S. Wycech, 2004 Int. J. Mod. Phys. E 13, 343.

[68] Bates Laboratory at MIT, http://bates.mit.edu/

[69] B. K. Agrawal, J. N. De, and S. K. Samaddar, 2012 Phys. Rev. Lett. 109, 262501.

[70] N. Nikolov, N. Schunck, W. Nazarewicz, M. Bender, and J. Pei, 2011 Phys. Rev. C 83, 034305.

[71] Mo Qiu Hong, Liu Min, Cheng Li Chun, and Wang Ning, 2015 Sci China-Phys Mech Astron 58, 082001.

[72] M. Bhuyan and S. K. Patra, 2012 Mod. Phys. Lett. A 27, 1250173; S. K. Patra, Raj K. Gupta and and W. Greiner, 1997 Mod. Phys. Lett. A12, 1727.

[73] S. Ahmad, M. Bhuyan, and S. K. Patra, 2012 Int. J. Mod. Phys. E 21, 1250092. 